\title{
An analysis of paediatric snakebites in north-eastern South Africa
}

\author{
JJP Buitendag, ${ }^{1}$ (i) S Variawa, ${ }^{2}$ (D) D Wood, ${ }^{3}$ (D) G Oosthuizen ${ }^{4}$ (D) \\ ${ }^{1}$ Department of Surgery, Tygerberg Hospital, Stellenbosch University, South Africa \\ ${ }^{2}$ Department of Surgery, Khayelitsha District Hospital, Stellenbosch University, South Africa \\ ${ }^{3}$ Department of Emergency Medicine, Queens Hospital BHRUT, Queen Mary University London, United Kingdom \\ ${ }^{4}$ Department of Surgery, Ngwelezana Hospital, University of KwaZulu-Natal, South Africa
}

Corresponding author, email: johan_buitendag@yahoo.com

Background: Snakebites are an underappreciated health concern in middle- and lower-income countries. The lack of national data vastly impacts funding for this health crisis, as well as strategies for treatment and prevention. Children are particularly vulnerable to snakebite and data in this group is limited.

Methods: This study included paediatric patients, aged 13 years old or younger, admitted to Ngwelezana Tertiary Hospital, Department of Surgery with a snakebite or snakebite related complication, from 1 September 2008 to 31 December 2013. Data captured included demographics, time of presentation, syndromic symptoms, blood results and patient management. Results: A total of 274 patients were included in this study. The median age at presentation was 8 years, with approximately $70 \%$ of the patients aged between 6 and 13 years, with a male predominance $(56 \%)$. The median time of presentation after sustaining a snakebite was 7 hours (interquartile range 4-13 hours). The majority of patients (71\%) presented with cytotoxic manifestations. A total of 53 patients received antivenom of whom $25 \%$ suffered adverse reactions. Fifty-six patients underwent one or more procedures on their affected limbs. Three patients required admission to the intensive care unit; all were part of the cytotoxic group and received antivenom. There were no recorded mortalities.

Conclusion: The majority of snakebites are cytotoxic in nature. One-fifth of the paediatric population require antivenom and one-fifth require a surgical procedure post envenomation. Adverse effects post antivenom use are common but manageable. Prevention programmes are needed to help reduce this burden of disease and a nationwide snakebite registry is long overdue.

Keywords: paediatric, snakebite, antivenom

\section{Introduction}

Snakebites are an underappreciated health concern in lowerand middle-income countries (LMICs). ${ }^{1}$ Global data on snakebites are region-specific and very limited regarding number of admissions, morbidity and mortality rates. The lack of data vastly impacts funding for this health crisis, as well as strategies for treatment and prevention. ${ }^{2}$ Current data suggests that Asia carries the highest burden of disease with approximately 15 400-57 600 deaths per annum, while sub-Saharan Africa estimates are at 3 500-32 100 deaths per annum. ${ }^{3}$ These estimates are based on hospital records and surveys from the affected regions.

Snakebite occurrence is reported more regularly in developed countries, however, lower-income countries are more commonly affected by snakebites therefore posing difficulties in analysis of epidemiological data. ${ }^{4}$ The North American Snakebite Registry was established in 2013 and records data collected prospectively across 10 states in the United States of America. ${ }^{5}$ Whilst this registry is dependent on accurate reporting, data collected has already provided beneficial information regarding directed public education, as well as further insight into the clinical manifestations of different snake species aiding treating physicians in management. ${ }^{5}$
There is data that suggests the number of snakebite cases in sub-Saharan Africa is as high as 100000-500 000 per annum. ${ }^{6}$ A meta-analysis has recently showcased that it is difficult to determine the exact burden of disease in this region. ${ }^{7}$ It is reported that the highest incidence of snakebites in Southern Africa is in rural KwaZulu-Natal with an annual incidence of 28-96.5 per $100000 .^{8-10}$ Another study estimated that the number of cases per year is between 1 193-2 357. ${ }^{\circ}$

In the last five decades, multiple studies on snakebites have reported similar findings on seasonal preference, demographics and mortality rates, which reiterates the fact that little progress has been made regarding treatment and prevention strategies for this underappreciated health concern..$^{8-10}$

The majority of potentially lethal snakebites in Southern Africa is due to cytotoxic envenomation from puff adders and the Mozambique spitting cobra. ${ }^{11}$ Due to the fact that snakebites and their outcomes are infrequently reported in Southern Africa, the mortality of 0.08-2.67 per 100000 is based on small studies. ${ }^{12}$ Children are more likely to be envenomated following a snakebite and suffer more severe presentations. This may often lead to a higher proportion of children receiving antivenom. ${ }^{13}$ The data on paediatric snakebites is scarce. Campbell et al. from Arkansas 
Children's Hospital and the University of Arkansas for Medical Sciences, published a paper with 114 cases of paediatric snakebites. ${ }^{14}$ Aktar et al. included 151 paediatric snakebites in a study performed by Dicle University in Turkey. ${ }^{15}$ Here we present the largest paediatric snakebite case series published, to our knowledge.

\section{Methods}

Ngwelezana Hospital is a 554-bed hospital, designated as a tertiary hospital for the northern part of the province of KwaZulu-Natal. The hospital receives referrals from 18 hospitals for secondary and tertiary care. The emergency department sees approximately 3500 patients annually.

\section{Sampling}

The patient population were all patients attending the emergency department at Ngwelezana Tertiary Hospital with snakebite from 1 September 2008 to 31 December 2013. The sample population included all patients aged 13 years old or younger who presented with snakebite during the specified period. As per national standard, the definition of a paediatric patient is considered as anyone below the age of 14 years. ${ }^{16}$ Patients were recruited consecutively and all patients in the study sample were included.

\section{Patient selection and management procedure}

Following admission, the condition of each snakebite patient was monitored from the time of admission to discharge by the surgical team. The diagnosis was made through a two-stage process. Firstly, if the patient identified the snake, brought it along to the hospital or visible fang marks were present, they were considered as having a suspected snakebite.

Secondly, suspected snakebite cases were further confirmed based on clinical symptoms on presentation such as local swelling, bleeding, or neurological symptoms. This system of syndromic assessment for snakebite is used to categorise envenomations and guide treatment. If the abovementioned criteria were not met, the patient was excluded from the study. Patients who developed significant swelling, bleeding or neurological symptoms were administered antivenom as per department protocol (Table I). Patients who required surgical intervention for skin necrosis had a procedure done in the operating theatre only once the inflammation had settled. The affected soft tissue damage was usually clearly demarcated after 3 to 5 days. Patients with significant limb swelling and suspected compartment syndrome were acted on without delay.

\section{Data}

The demographic and clinical data of the patient was captured on a case report form and entered onto a secured database (Excel 2002). Data fields included patient demographics, time of bite to presentation, syndromic clinical symptoms, blood results, patient management and outcome. Ethical permission for the study was approved by the University of KwaZulu-Natal Biomedical Research Ethics Committee (Ref. No.: BE034/14)

\section{Statistical analysis}

Descriptive statistics were initially done to describe the basic features of the data at hand and to indicate the dispersion of the data. These descriptive methods were also used to indicate the skewness and kurtosis of the data. In cases where outliers were evident, rank and percentile tests were

Table I: Protocol for antivenom administration
1. Indication correct - clinical severity, risk (SRS), pre-surgery
2. Consent from patient or guardian
3. Transfer patient to resuscitation area
4. Full monitoring
5. 2 x IV lines (large bore) appropriate for age
- 1 x Crystalloid for resuscitation
- 1 x Antivenom
6. Premedication
- Adrenaline IM
- $0.3-0.5 \mathrm{mg}$ in adults
- $0.01 \mathrm{mg} / \mathrm{kg}(1: 1000$ ) or $0.1 \mathrm{ml} / \mathrm{kg}$ ( $1: 10000)$ in children
- Expect tachycardia, monitor for dysrhythmias
- Fluid bolus $20 \mathrm{ml} / \mathrm{kg}$
7. Antivenom initial dose
- 5 vials polyvalent for painful progressive swelling (repeat
as required)
- 10 vials polyvalent for neurotoxic progressive weakness
- 2 vials monovalent Boomslang in bleeding
- Dilute in $200 \mathrm{ml}$ normal saline and infuse over $20-30$
minutes. (test dose not required)
8. Repeat doses of antivenom prn
9. Dr to closely monitor patient
10. Patients to be counselled re: Serum (type III reaction)
sickness

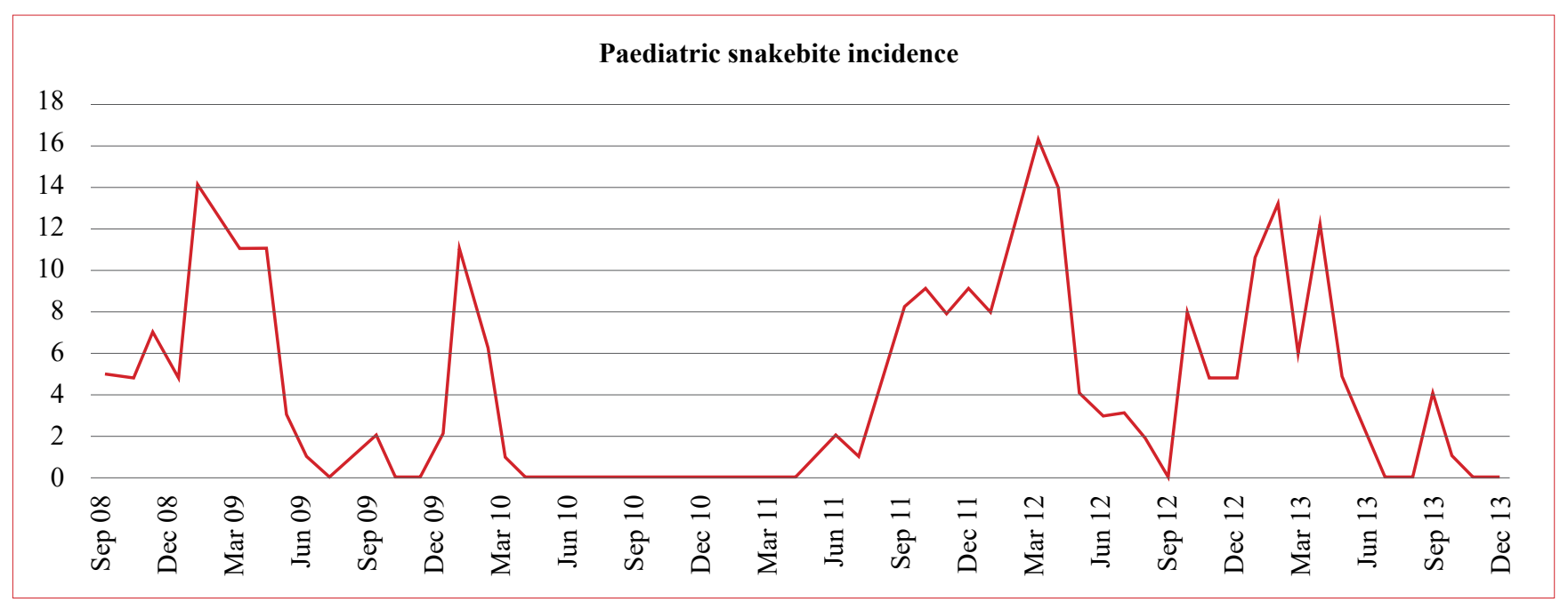

Figure 1: Paediatric snakebite monthly incidence 
done to measure the true dispersion. Descriptive analysis was also used in aid of trend analysis of the historic data to make meaningful inferences. Mortality rate, age and sex are commonly measured with this method.

Continuous variables were compared using unpaired $t$-test, and categorical variables using chi-squared analysis. Chi-squared test compared the type of envenomation and subsequent treatment and reactions. Chi-squared analysis was also used to assess other categorical variables between groups.

\section{Results}

\section{Demographics}

Over a 5-year period from 1 September 2008 to 31 December 2013, a total of 274 children aged 13 years or younger presented with a snakebite. The median age at presentation was 8 years, with approximately $70 \%$ of the patients aged 6-13 years. Forty-four per cent of patients were female and $56 \%$ male. The majority of the patients were from the immediate area with $24 \%$ of patients referred from surrounding facilities. The peak incidence occurred over the warmer months, which are usually December-February (Figure 1).

\section{Time after bite}

The median time of presentation to Ngwelezana Hospital after sustaining a snakebite was 7 hours with an IQR of 4-13 hours. The earliest presentation was after 30 minutes and latest after 79 hours.

\section{Syndromic distribution}

The vast majority of patients (71\%) presented with cytotoxic manifestations, $2 \%$ haemotoxic and $1 \%$ neurotoxic, while $26 \%$ of patients displayed no features in keeping with any of the latter categories and are unclassified in the data. Four patients presented with signs of both cytotoxic and haemotoxic manifestations and one patient with cytotoxic and neurotoxic manifestations. Patients with mixed envenomation were classified according to the predominant syndrome present.

\section{Blood results}

For the total patient sample, the median white cell count (x 109/L) was 10.0 , haemoglobin (g/dL) 11.8 , platelets (x $10^{9} / \mathrm{L}$ ) 308, INR (international normalised ratio) 1.12, urea (mmol/L) 3.3 and creatinine (umol/L) 43 (Table II). In the cytotoxic group, the median white cell count was 10.45 $( \pm 6.3)$, haemoglobin $11.7( \pm 2.1)$, platelets $301( \pm 146)$, INR $1.13( \pm 0.3)$, urea $3.3( \pm 1.6)$ and creatinine $42( \pm 18)$. In the haemotoxic group, the median white cell count was 14.0 $( \pm 5.3)$, haemoglobin $7.8( \pm 3.8)$, platelets $324( \pm 163)$, INR $3.82( \pm 4.0)$, urea $2.4( \pm 3.4)$ and creatinine $28( \pm 56)$. In the neurotoxic group, the median white cell count was 15.9 $( \pm 2.6)$, haemoglobin $8.45( \pm 3.1)$, platelets $142( \pm 122)$, INR $1.28( \pm 0)$, urea $3.8( \pm 1.8)$ and creatinine $39( \pm 9)$. Six patients suffered an acute kidney injury (AKI) according to the Kidney Disease - Improving Global Outcomes (KDIGO) classification system (creatinine $\geq 1.5 \mathrm{x}$ upper limit of normal) of which five were classified as cytotoxic and the patient with the most severe AKI was classified as haemotoxic. Two patients with an AKI received antivenom, however, both did not develop signs of anaphylactic shock. None of the patients with an AKI developed compartment syndrome or required limb amputation.

\section{Management}

A total of 53 out of 274 patients (19\%) received antivenom of which 45 were categorised as cytotoxic, six were haemotoxic and two were neurotoxic. On average, six vials of antivenom were administered. Antivenom was administered to $23 \%$ of patients with cytotoxic presentations and $100 \%$ of patients with haemotoxic and neurotoxic presentations respectively. Of the total number of patients who received antivenom, 25\% suffered adverse reactions. Six patients developed allergic reactions of which one patient progressed to anaphylaxis and seven patients developed anaphylaxis. Allergic reactions were considered as mild skin manifestations, e.g., skin rash or urticaria without systemic involvement. Anaphylactic reactions were considered as a drop in blood pressure from baseline, central nervous system deterioration, impalpable radial pulse, signs of airway obstruction and haemodynamic instability, with or without skin manifestations. All patients with hypersensitivity reactions received vasopressor support in the form of adrenaline infusion as well as intravenous fluid administration. One patient subsequently developed

Table II: Admission bloods overview

\begin{tabular}{|c|c|c|c|c|c|c|}
\hline \multicolumn{7}{|c|}{ Admission bloods overview } \\
\hline & WCC $\left(\times 10^{9} / L\right)$ & $\mathrm{Hb}(\mathrm{g} / \mathrm{dL})$ & Platelets $\left(\times 10^{9} / \mathrm{L}\right)$ & INR & Urea $(\mathrm{mmol} / \mathrm{L})$ & Creatinine (umol/L) \\
\hline Min & 1.38 & 5.40 & 13.00 & 0.90 & 0.9 & 15 \\
\hline Q1 & 7.93 & 10.60 & 237.00 & 1.04 & 2.5 & 34 \\
\hline Q2 & 10.00 & 11.80 & 308.00 & 1.12 & 3.3 & 43 \\
\hline Q3 & 13.85 & 12.80 & 379.00 & 1.28 & 4.0 & 53 \\
\hline Max & 26.10 & 15.70 & 987.00 & 7.10 & 41.0 & 688 \\
\hline Mean & 11.17 & 11.59 & 319.56 & 1.30 & 3.8 & 48 \\
\hline Median & 10.00 & 11.80 & 308.00 & 1.12 & 3.3 & 43 \\
\hline Range & 24.72 & 10.30 & 974.00 & 6.20 & 40.1 & 673 \\
\hline IQR & 5.93 & 2.20 & 142.00 & 0.24 & 1.5 & 19 \\
\hline Std dev & 4.62 & 1.73 & 139.90 & 0.69 & 3.86 & 48 \\
\hline
\end{tabular}

WCC - white cell count, $\mathrm{Hb}$ - haemoglobin, INR - international normalised ratio 
Table III: Procedures performed for children with snakebites

\begin{tabular}{lc}
\hline Cytotoxic & $\boldsymbol{n}(\mathbf{\%})$ \\
\hline Procedure & $28(10)$ \\
\hline Split skin graft & $48(18)$ \\
Debridement & $1(0.4)$ \\
Fasciotomy & $5(0.8)$ \\
Amputation & \\
Haemotoxic & $3(1.1)$ \\
\hline Split skin graft & $3(1.1)$ \\
Debridement & $1(0.4)$ \\
Fasciotomy & \\
Neurotoxic & $2(0.7)$
\end{tabular}

cardiovascular collapse and required cardiopulmonary resuscitation with return of spontaneous circulation. There was no correlation noted between age and the development of hypersensitivity reactions.

\section{Outcome}

A total of 56 patients underwent one or more procedures on their affected limbs; 32 patients had a split skin graft, 54 were debrided, two had a fasciotomy and five had belowknee amputations. The cytotoxic group accounted for the majority of procedures performed (Table III). The median time of presentation after bite for the five patients who required amputations was 10 hours (range 18.5 hours). Four of the five patients received antivenom and were below the age of 5 with a median age of 3 years. All patients who required lower limb amputation presented with progressive tissue necrosis and none developed compartment syndrome. Delay in presentation, and therefore antivenom administration, was considered as the most significant contributing factor for the need for limb amputation. Three patients aged 2, 9 and 13 required admission to the ICU of which all were part of the cytotoxic group and received antivenom. Only one of these patients suffered an adverse drug reaction (anaphylaxis), which was treated, and subsequently underwent a debridement and split skin graft of the affected limb. There was no correlation between age and admission to ICU. There were no recorded mortalities during this period.

\section{Discussion}

To date, this is the largest series of paediatric snakebites that we are aware of. Studies have shown that children under the age of 15 years are more vulnerable to sustaining snake bites due to inherent curiosity, lack of caution as well as walking barefoot. ${ }^{17}$ In rural areas, older children are often also involved in outdoor agricultural responsibilities. ${ }^{15,18}$ Wood et al. reported that children under 12 years were an independent risk factor for the severity of snakebites. ${ }^{19}$ A study by Campbell et al. in the United States of America reported on a series consisting of 114 cases and the lessons they learned. ${ }^{14}$ They reported a mean age of 7.3 years and males being more affected at 60\%; Arkanas et al. reported similar findings with a mean age of \pm 10 years. Kshirsagar et al. also reported a male predominance $(60.49 \%)$ with a mean age of 9.2 years in males and 8.6 years in females in rural India. ${ }^{20}$ Our data is in keeping with this trend with a mean age of 7.7 years and males accounting for $56 \%$ of cases. Interestingly they reported only $6 \%$ of patients requiring antivenom and only a single case had an anaphylactic reaction. ${ }^{12}$ In our series, $19 \%$ of patients required antivenom and eight had an anaphylactic reaction. Two of their patients required fasciotomies whereas in our data set, with significantly more patients, we also reported two fasciotomies. ${ }^{12}$ This might stem from our more liberal use of antivenom. In a study performed in a tertiary setting in Texas, United States, antivenom administration was more liberal (34\%) with only one (2\%) hypersensitivity reaction recorded. Of the 151 patients included in the study, only four $(2.6 \%)$ required surgical intervention. ${ }^{21}$ The antivenom most commonly administered in their setting was CroFab, specific to crotaline species, which has been shown to have a low rate of hypersensitivity reactions. ${ }^{21,22}$

The data on snakebites in Southern Africa is predominantly from the KwaZulu-Natal region and its draining hospitals. ${ }^{6-8,11}$ There are five main clinical syndromes of snake envenomation recognised in South Africa as described by Müller et al.:

- Marked local pain and progressive swelling associated with prominent cytotoxic skin changes with coagulable blood.

- $\quad$ Progressive paralysis (neurotoxicity), with negligible or minor local swelling.

- Incoagulable blood, with negligible to mild local swelling.

- Moderate to marked local swelling, associated with neurotoxicity.

- Mild to moderate swelling, with negligible or absent systemic symptoms. ${ }^{12}$

Since there is overlapping of these syndromes, in our data series, we categorised our patients into cytotoxic, haemotoxic, neurotoxic and unclassified.

Wood et al. reported that $98 \%$ of their admissions in adults were due to cytotoxic envenomation. ${ }^{11}$ They reported four cases of haemotoxicity and five cases of neurotoxicity that were admitted. ${ }^{13}$ In our series $71 \%$ presented with cytotoxic manifestations, $2 \%$ haemotoxic and $1 \%$ neurotoxic, while $26 \%$ of patients displayed no features in keeping with any of the latter categories. The similarities to the study conducted by Wood et al. is evident. They also reported that $1 / 3(33 \%)$ snakebites will have no envenomation symptoms whereas our data series had $26 \%$ patients with no symptoms of envenomation.

According to a study conducted in north-eastern Nigeria, the risk of mortality secondary to envenomation increases by $1 \%$ for every 1 -hour delay between the time of the bite and presentation to medical personnel. ${ }^{23}$ Kshirsagar et al. reported a mortality rate of $1.85 \%$ in a study evaluating paediatric snakebites in rural areas of Maharashtra, India. All patients who demised presented to the hospital $>8$ hours after the time of the bite. ${ }^{20}$ Transport limitations, presentation to traditional healers prior to seeking conventional medical treatment and increasing distance from healthcare facilities may play a role in delayed presentation. ${ }^{18,24}$ In our study, the median time of presentation to the hospital after the time of the bite was 7 hours with an IQR of 9 hours compared to 1.5 hours in an urban area in Taiwan. ${ }^{25}$ This may be attributed to the fact that many of the patients in our study come from surrounding rural areas that do not have access to transport which may contribute to the delayed presentation. This may 
increase the risk for severe envenomation and therefore mortality.

In our study, the INR median was $1.13( \pm 0.3)$ in the cytotoxic group, $3.82( \pm 4)$ in the haemotoxic group and $1.28( \pm 0)$ in the neurotoxic group. The median INR is significantly higher in the haemotoxic group as expected; however, due to the low number of haemotoxic (6) and neurotoxic (2) patients it is difficult to draw a definitive conclusion. The median haemoglobin was also significantly lower $(<11 \mathrm{~g} / \mathrm{dL})$ in the haemotoxic group followed by the neurotoxic group. This finding is in keeping with the results reported by Hernandez et al. ${ }^{26}$ The median white cell count was highest within the neurotoxic group. Of note is that there was a low rate $(2.2 \%)$ of acute kidney injury in our study. These occurred mostly within the cytotoxic group and one within the haemotoxic group. In comparison to a study conducted amongst adults in India, the rate of AKI was significantly higher $(21.1 \%)$. Similarly, the predominating syndromes associated were cytotoxic and haemotoxic. ${ }^{27}$

Isbister et al. reported a study in which antivenom was most administered due to presence of coagulopathy followed by non-specific manifestations and neurotoxicity. In this study, $25 \%$ of patients who received antivenom suffered from hypersensitivity reactions, of which $44 \%$ were classified as anaphylaxis. ${ }^{28}$ In comparison to our study, of the 53 patients who received antivenom, 45 suffered cytotoxic envenomation equating to $23 \%$ of all patients with cytotoxic envenomation as compared to $100 \%$ of haemotoxic and neurotoxic patients who received antivenom. In comparison to the study conducted by Isbister et al., hypersensitivity reactions were noted in $23 \%$ of our patients who received antivenom, of which $62 \%$ were classified as anaphylaxis.

Hernandez et al. reported a study analysing the surgical considerations in paediatric snake bites conducted at Pietermaritzburg Metropolitan Trauma Service. ${ }^{26}$ This study included 72 patients below the age of 18 years of which a total of nine patients $(12.5 \%)$ received antivenom and two subsequently developed anaphylaxis and were admitted to ICU. In total, ten patients $(14 \%)$ were admitted to ICU of which seven were classified as cytotoxic ( $11 \%$ of all cytotoxic admissions), one neurotoxic ( $20 \%$ of neurotoxic admissions) and two haemotoxic (50\% of haemotoxic admissions). ${ }^{26}$ In comparison to our study in a similar setting, $19 \%$ of patients received antivenom and a total of three patients $(1.1 \%)$ required ICU admission for further monitoring following the administration of antivenom due to severe envenomation of which only one patient developed anaphylaxis and required surgical intervention to the affected limb. All three patients suffered from cytotoxic envenomation.

Hernandez et al. reported $5.5 \%$ of patients requiring subsequent debridement compared to $20 \%$ of the patients included in our study. ${ }^{26}$ Seven per cent of patients had a split skin graft at their facility compared to $11 \%$ at Ngwelezana Hospital. They also report 18 fasciotomies (25\%) done as compared to two fasciotomies performed at Ngwelezana Hospital (0.7\%). ${ }^{17}$ This may be explained by the higher number of patients who received antivenom at Ngwelezana Hospital as well as less delay in presentation. This is supported by the median time of presentation after injury at Pietermaritzburg of 20 hours compared to 7 hours at Ngwelezana Hospital. ${ }^{26}$ Only one amputation was reported at Pietermaritzburg ( $1.4 \%$ incidence) compared to five amputations performed at Ngwelezana Hospital
(1.8\% incidence).$^{26}$ There was an increase in median time of presentation after bite of 10 hours in the group of patients who had amputations compared to the overall sample population. Interestingly, four of the five patients who underwent amputations were below the age of 5. Severe envenomation is more likely in children due to their smaller body mass and this can explain the need for amputation in these very young children. Amputations pose a detrimental impact on the psychological and physical well-being of children. The ongoing growth within the residual limb and abnormal residual stump shape also poses an additional challenge with prosthetic rehabilitation. ${ }^{29}$ Approximately $89 \%$ of procedures performed at Ngwelezana Hospital were for patients with cytotoxic envenomation, including all five amputations.

In a study comparing snakebites between the paediatric and adult populations, Tekin et al. reported a mortality rate of $0.8 \%$ amongst children. ${ }^{30}$ There were no recorded mortalities in our study. This may be an indicator of effectiveness of our current management principles.

\section{Conclusion}

Paediatric snakebites are a significant burden on the health system of Southern Africa. Most snakebites in paediatric patients are cytotoxic in nature. As much as one-fifth (20\%) of the paediatric population may require antivenom as well as a surgical procedure post envenomation. Adverse effects post antivenom use are common but manageable without risk of major morbidity or mortality. Snakebites in this population subgroup should be deemed as severe and extreme caution should be exercised when administering antivenom.

Prevention programmes are needed to help reduce this burden of disease and a nationwide snakebite registry is long overdue.

\section{Conflict of interest}

The authors declare no conflict of interest.

\section{Funding source}

None.

\section{Ethical approval}

Ethical approval for the study was approved by the University of KwaZulu-Natal Biomedical Research Ethics Committee (Ref. No.: BE034/14).

\section{ORCID}

JJP Buitendag (iD https://orcid.org/0000-0001-7169-129X

S Variawa iD https://orcid.org/0000-0001-7672-1259

D Wood (D) https://orcid.org/0000-0002-3151-5651

G Oosthuizen (iD https://orcid.org/0000-0002-6936-5716

\section{REFERENCES}

1. Gutiérrez JM. Improving antivenom availability and accessibility: science, technology, and beyond. Toxicon. 2012;60(4):676-87.https://doi.org/10.1016/j.toxicon.2012.02. 008 .

2. Kasturiratne A, Wickremasinghe AR, De Silva N, et al. The global burden of snakebite: a literature analysis and modelling based on regional estimates of envenoming and deaths. PLoS 
Med. 2008;5(11):e218. https://doi.org/10.1371/journal.pmed. 0050218.

3. Harrison RA, Hargreaves A, Wagstaff SC, Faragher B, Lalloo DG. Snake envenoming: a disease of poverty. PLoS Negl Trop Dis. 2009;3(12):e569. Published 2009 Dec 22. https:// doi.org/10.1371/journal.pntd.0000569.

4. De la O Cavazos ME, Treviño Garza C, Guajardo-Rodríguez G, Hernández-Montelongo BA, Montes-Tapia FF. Snake bites in pediatric patients, a current view. Complementary Pediatrics, IntechOpen; 2012. https://doi.org/10.5772/34749.

5. Ruha AM, Kleinschmidt KC, Greene S, et al.; on behalf of the ToxIC Snakebite Study Group. The epidemiology, clinical course, and management of snakebites in the North American Snakebite Registry. J Med Toxicol. 2017;13(4),309-20. https://doi.org/10.1007/s13181-017-0633-5.

6. Chippaux JP. Snake-bites: appraisal of the global situation. Bull World Health Organ. 1998;76(5):515-24.

7. ChippauxJP.Estimate of the burden of snakebitesinsub-Saharan Africa: a meta-analytic approach. Toxicon. 2011;57(4):58699. https://doi.org/10.1016/j.toxicon.2010.12.022.

8. Blaylock R. Epidemiology of snakebite in Eshowe, KwaZuluNatal, South Africa. Toxicon. 2004;43(2):159-66. https://doi. org/10.1016/j.toxicon.2003.11.019.

9. Sloan DJ, Dedicoat MJ, Lalloo DG. Healthcare-seeking behaviour and use of traditional healers after snakebite in Hlabisa sub-district, KwaZulu-Natal. Trop Med Int Health. 2007;12(11):1386-90. https://doi.org/10.1111/j.1365-3156. 2007.01924.x.

10. Wilkinson D. Retrospective analysis of snakebite at a rural hospital in Zululand. S Afr Med J. 1994;84(12):844-7.

11. Darryl W, Sartorius B, Hift R. Estimating the burden of snakebite on public hospitals in KwaZulu-Natal, South Africa. Wilderness Environ Med. 2016;27(1):53-61. https:// doi.org/10.1016/j.wem.2015.11.005.

12. Müller GJ, Modler H, Wium CA, et al. Snake bite in southern Africa: diagnosis and management. Continuing Med Edu. 2012;30(10):362-82.

13. Wood D, Sartorius B, Hift R. Snakebite in north-eastern South Africa: clinical characteristics and risks for severity, South African Family Practice. 2016;1(1):1-6. https://doi.org/10.10 80/20786190.2015.1120934.

14. Campbell BT, Corsi JM, Boneti C, et al. Pediatric snakebites: lessons learned from 114 cases. J Pediatr Surg. 2008;43(7):1338-41. https://doi.org/10.1016/j.jpedsurg.2007. 11.011 .

15. Aktar F, Aktar S, Yolbas I, Tekin R. Evaluation of risk factors and follow-up criteria for severity of snakebite in children. Iran J Pediatr. 2016;26(4):e5212. https://doi.org/10.5812/ijp.5212.

16. Republic of South Africa. Age definitions. Republic of South Africa. National Department of Health; 2012. Available from: http://www.health.gov.za/index.php/shortcodes/201503-29-10-42-47/2015-06-10-09-23-36/2015-06-10-09-2611 ?download $=844$ :icd-10-definitions\&start $=15$.
17. Shrestha B. Outcomes of snakebite envenomation in children. Journal of Nepal Paediatric Society. 2011;31(3):192-7. https:// doi.org/10.3126/jnps.v31i3.5358.

18. Meshram R, Bokade C, Merchant S, Bhongade S. Clinical profile and outcome of snake bite in children. Int J Contemp Pediatrics. 2017;4(3):910-4. https://doi.org/10.18203/23493291.ijcp20171697.

19. Wood D, Sartorius B, Hift R. Classifying snakebite in South Africa: validating a scoring system. South African Medical Journal. 2017;107(1):46-51. https://doi.org/10.7196/ SAMJ.2017.v107i1.11361.

20. Kshirsagar VY, Ahmed M, Colaco SM. Clinical profile of snake bite in children in rural India. Iran $\mathrm{J}$ Pediatr. 2013;23(6):632-6.

21. Schmidt JM. Antivenom therapy for snakebites in children: is there evidence? Curr Opin Pediatr. 2005;17(2):234-8. https:// doi.org/10.1097/01.mop.0000152621.67049.f2.

22. Correa JA, Fallon SC, Cruz AT, et al. Management of pediatric snake bites: are we doing too much? J Pediatr Surg. 2014;49(6):1009-15. https://doi.org/10.1016/j.jpedsurg.2014 01.043 .

23. Habib AG, Abubakar SB. Factors affecting snakebite mortality in north-eastern Nigeria. Int Health. 2011;3(1):50-5. https:// doi.org/10.1016/j.inhe.2010.08.001.

24. Ndu IK, Edelu B, Ekwochi U. Snakebites in a Nigerian children population: a 5-year review. Sahel Medical Journal. 2018;21:204-7. https://doi.org/10.4103/smj.smj_18_18.

25. Wang JD, Tsan YT, Yan-Chiao M, Wang LM. Venomous snakebites and antivenom treatment according to a protocol for pediatric patients in Taiwan. J Venom Anim Toxins Incl Trop Dis. 2009;15(4):667-79. https://doi.org/10.1590/S167891992009000400006.

26. Hernandez MC, Traynor M, Bruce JL, et al. Surgical considerations for pediatric snake bites in low- and middleincome countries. World J Surg. 2019;43(7):1636-43. https:// doi.org/10.1007/s00268-019-04953-9.

27. George TK, Toms AG, Fenn BN, et al. Renal outcomes among snake-envenomed patients with acute kidney injury in southern India. Natl Med J India. 2019;32(1):5-8. https://doi. org/10.4103/0970-258X.272106.

28. Isbister GK, Brown SG, MacDonald E, White J, Currie BJ; Australian Snakebite Project Investigators. Current use of Australian snake antivenoms and frequency of immediatetype hypersensitivity reactions and anaphylaxis. Med J Aust. 2008;188(8):473-6.

29. Eshraghi A, Safaeepour Z, Geil MD, Andrysek J. Walking and balance in children and adolescents with lower-limb amputation:Areview of literature. Clin Biomech. 2018;59:18198. https://doi.org/10.1016/j.clinbiomech.2018.09.017.

30. Tekin R, Sula B, Cakırca G, et al. Comparison of snakebite cases in children and adults. Eur Rev Med Pharmacol Sci. 2015;19(14):2711-6. 